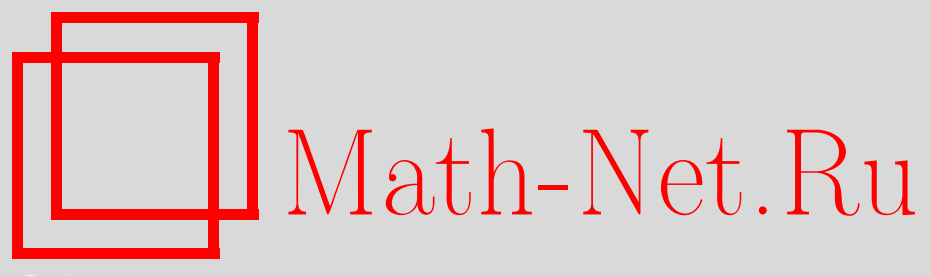

Н. Ю. Ероховец, Инвариант Бухштабера простых многогранников, УМH, 2008, том 63, выпуск 5, 187-188

DOI: https://doi.org/10.4213/rm9231

Использование Общероссийского математического портала Math-Net.Ru подразумевает, что вы прочитали и согласны с пользовательским соглашением http://www . mathnet.ru/rus/agreement

Параметры загрузки:

IP: 52.6 .47 .48

26 апреля 2023 г., $14: 27: 41$

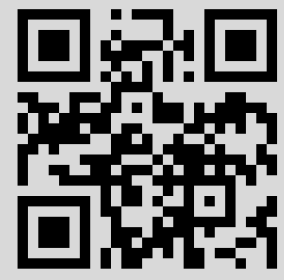




\section{Инвариант Бухштабера простых многогранников}

\section{Н. Ю. Ероховец}

Пусть $P=\left\{x \in \mathbb{R}^{n}: A_{p} x \leqslant b_{p}\right\}$ - простой многогранник и $\mathfrak{F}=\left\{F_{1}, \ldots, F_{m}\right\}-$ множество его гиперграней. Каждой грани $F_{i} \in \mathfrak{F}$ поставим в соответствие одномерную координатную подгруппу в $T^{\mathfrak{F}}=T^{m}$. Грани $G$ многогранника $P$ сопоставим координатную торическую подгруппу $T^{G}=\prod_{F_{i} \supset G} T^{F_{i}} \subset T^{\mathfrak{F}}$. Для каждой точки $q \in P$ обозначим через $G(q)$ наименьшую грань, содержащую $q$.

Для комбинаторного простого многогранника $P^{n}$ положим: $\mathscr{Z}_{P}=\left(T^{\mathfrak{F}} \times P^{n}\right) / \sim$, где $\left(t_{1}, p\right) \sim\left(t_{2}, q\right)$ тогда и только тогда, когда $p=q$, и $t_{1} t_{2}^{-1} \in T^{G(p)}$.

Оказывается, $\mathscr{Z}_{P}$ является гладким многообразием размерности $m+n$ с гладким действием тора $T^{m}$, индуцированным стандартным действием тора на первом сомножителе. При этом $\mathscr{Z}_{P} / T^{m}=P$, а стабилизатором точки $[(t, q)]$ является $T^{G(q)}$.

ОПРедЕЛЕНИЕ. Числом Бухштабера $s(P)$ называется максимальная размерность nодгрупп $H \cong T^{s}$, действующих свободно.

Проблема, поставленная В.М. Бухштабером в 2002 г. заключается в том, чтобы научиться конструктивно вычислять $s(P)$ в комбинаторных терминах.

Заметка посвящена изучению свойств инварианта $s(P)$.

Было известно, что $1 \leqslant s(P) \leqslant m-n$ и $s(P)=1 \Leftrightarrow P=\Delta^{n}$. И. В. Изместьев [1] заметил, что $s(P) \geqslant m-\gamma(P)$, где $\gamma(P)$ - хроматическое число многогранника $P$ (наименьшее число цветов, в которые можно раскрасить гиперграни так, что соседние будут разных цветов).

Далее мы используем следующее описание $s(P)$ (см. [2; лемма 8.24]): в выбранном базисе $T^{m}$ торической подгруппе $H$ размерности $s$ соответствует целочисленная матрица $A$ размера $m \times s$. Действие $H$ свободно тогда и только тогда, когда для любой вершины подматрица размера $(m-n) \times s$, состоящая из всех строк матрицы $A$, соответствующих гиперграням $P$, не проходящим через эту вершину, задает вложение $\mathbb{Z}^{s} \rightarrow \mathbb{Z}^{m-n}$ на прямое слагаемое. Если дополнить отображение, определяемое $A$, до точной последовательности: $0 \longrightarrow \mathbb{Z}^{s} \stackrel{A}{\longrightarrow} \mathbb{Z}^{m} \stackrel{B}{\longrightarrow} \mathbb{Z}^{m-s} \longrightarrow 0$, то мы получим еще один вариант определения $s(P)$ - это наибольшее натуральное число, при котором существует отображение $\mathfrak{F} \rightarrow \mathbb{Z}^{m-s}$ такое, что для любой вершины образ набора гиперграней, пересекающихся в этой вершине, можно дополнить до базиса целочисленной решетки $\mathbb{Z}^{m-s}$. Заметим, что при $s=m-n$ это в точности условие $*$ Дена-Соммервилля [2]. Для любого $(n-1)$-мерного симплициального комплекса $K$ имеются соответствующие определения $s(K)$ такие, что $s\left(\partial\left(P^{*}\right)\right)=s(P)$.

Теорема. Число $s(P)$ обладает следующими свойствами:

1) $s(P)+s(Q) \leqslant s(P \times Q) \leqslant s(P)+s(Q)+\min \left(m_{1}-n_{1}-s(P), m_{2}-n_{2}-s(Q)\right)$;

2) $s(P \sharp Q) \geqslant s(P)+s(Q)$, где $P \sharp Q-$ связная сумма многогранников вдоль вершин;

3) $s(P) \geqslant s(Q)$, если $Q$ получается из $P$ забыванием одного из $m$ неравенств;

4) $s(F) \geqslant m_{1}-m+s(P)$, где $F$ - гипергрань многогранника с $m_{1}$ гипергранями;

5) $s(P)$ нельзя вычислить, зная только $f$-вектор многогранника и $\gamma(P)$;

6) для разветвленного комбинаторного накрытия (см. [3]) $\partial\left(P^{*}\right) \rightarrow K$ грании,ы двойственного многогранника над симплициальным комплексом с $k$ вершинами $s(P) \geqslant m-k+s(K)$;

7) $s(P) \geqslant m-\gamma(P)+s\left(\Delta_{n-1}^{\gamma-1}\right)$, где $\Delta_{n-1}^{\gamma-1}-$ это $(n-1)$-мерный остов $(\gamma-1)$-мерного симплекса;

8) если $\widehat{P}$ получается из $P$ движением гиперграни таким, что до некоторого момента времени комбинаторный тип многогранника не изменяется, а затем движущаяся гиперплоскость отсекает ровно одну новую вершину, то $|s(P)-s(\widehat{P})| \leqslant 1$. 
Последняя операция является бизвездным преобразованием симплициального комплекса $\partial\left(P^{*}\right)$. Эти преобразования были введены У. Пахнером для исследования свойств PL-многообразий. Таким образом, если ввести на пространстве простых многогранников метрику $d(P, Q)$ - наименьшее число бизвездных преобразований, переводящих $\partial\left(P^{*}\right)$ в $\partial\left(Q^{*}\right)($ см. [4]), то из свойства 8) следует, что $|s(P)-s(Q)| \leqslant d(P, Q)$.

Чтобы получить свойства 1$)-3)$, удобно использовать первый вариант комбинаторного определения $s(P)$, а для доказательства свойств 4) и 5) больше подходит второй.

Свойства 6), 7) можно установить, исследуя свойства комбинаторных разветвленных накрытий (т. е. невырожденных симплициальных отображений): правильная раскраска в $\gamma$ цветов гиперграней многогранника $P$ эквивалентна невырожденному симплициальному отображению $\partial\left(P^{*}\right) \rightarrow \Delta_{n-1}^{\gamma-1}$, а при таком отображении можно индуцировать отображения вершин в $\mathbb{Z}^{m-s}$.

Укажем пример двух многогранников с одинаковыми $f$-векторами и хроматическими числами, но разными $s(P)$. Нам потребуется описание простого $n$-многогранника с не более чем $n+3$ гипергранями [5] в терминах правильного $(2 k-1)$-угольника, $k \geqslant 2$, у которого в вершинах расставлены числа $a_{1}, \ldots, a_{2 k-1}, a_{i}>0, \sum a_{i}=n+3$. Каждой гиперграни соответствует вершина многоугольника, причем три такие гиперграни задают вершину $P$, в которой пересекаются оставшиеся $n$ тогда и только тогда, когда 0 лежит в треугольнике, образованном соответствующими вершинами.

УтвеРЖДЕНИЕ 1 . Если $m=n+3$, mo $s(P)=3$ тогда и только тогда, когда $k \leqslant 4$.

Бизвездное преобразование переводит многогранник $\left(a_{1}, \ldots, a_{i}, \ldots, a_{i+k}, \ldots, a_{2 k-1}\right)$ в многогранник $\left(a_{1}, \ldots, a_{i}-1,1, a_{i+1}, \ldots, a_{i+k-1}, 1, a_{i+k}-1, \ldots, a_{2 k-1}\right)$, при этом если $a_{s}$ становится равным 0 , нужно объединить в одну вершину точки противоположного $a_{s}$ ребра, а числа сложить.

Из этого наблюдения и того, как изменяется $h$-вектор при бизвездном преобразовании (см. [2]), получаем утверждение

УТВеРЖДЕНИЕ 2. Если $m \leqslant n+3$, mo

$$
h(P)=(t-1)^{-3}\left(t^{n+3}-1+\sum_{i=1}^{2 k-1} t^{a_{i}+\cdots+a_{i+k-2}}-\sum_{i=1}^{2 k-1} t^{a_{i}+\cdots+a_{i+k-1}}\right) .
$$

Рассмотрим два многогранника: $P=(2,1,1,1,1,1,1,1,1)$ и $Q=(2,1,2,1,1,2,1)$. Многогранник $P$ является двойственным к циклическому, а $Q$ получается из него бизвездным 3-преобразованием, не меняющим $f$-вектор и $\gamma(P)$. Но $s(P)=2, s(Q)=3$.

Свойство 8) можно получить из комбинаторного описания перехода через вершину: имеется $n+1$ гипергрань такая, что для любой из первых $i$ гиперграней оставшиеся $n$ задают вершину, а для любой из гиперграней с номерами $i+1, \ldots, i+j=n+1$ не задают. Тогда бизвездное преобразование меняет местами эти множества, и если во втором определении $s(P)$ каждому вектору $\mathbb{Z}^{m-s}$, соответствующему всем гиперграням, кроме одной из первого множества, добавить в конце 0 , а оставшемуся вектору добавить в конце 1 , то мы увидим, что $s(\widehat{P}) \geqslant s(P)-1$.

Автор благодарен В. М. Бухштаберу за постановку задачи и постоянное внимание к работе.

\section{Список литературы}

[1] И. В. Изместьев, Матем. заметки, 69:3 (2001), 375-382. [2] В. М. Бухштабер, Т. Е. Панов, Торические действия в топологии и комбинаторике, МЦНМО, М., 2004. [3] В. М. Бухштабер, Т. Е. Панов, Тр. МИАН, 247, Наука, М., 2004, 41-58. [4] A. Nabutovsky, Comm. Math. Phys., 181:2 (1996), 303-330. [5] B. Grünbaum, Convex polytopes, Wiley, New York, 1967.

Н. Ю. Ероховец (N. Yu. Erokhovets)

Московский государственный университет им. М. В. Ломоносова

E-mail: erochovetsn@hotmail.com
Представлено В. М. Бухштабером Принято редколлегией 09.06.2008 